\title{
Lymphangioma Circumscriptum with Features of both Classic and Localized Forms Treated with Carbon Dioxide Laser
}

\section{Santos MK*, Lopez-Villafuerte L and Doria-Ruiz M}

Jose R. Reyes Memorial Medical Center Department of Dermatology, Philippines

*Corresponding author: Marissa Kathrina Santos MD, Resident, Jose R. Reyes Memorial Medical Center Department of Dermatology, Philippines, Tel: (02)711-9491

loc 336; Email: marissakathrinasantos@yahoo.com

\section{Case Report}

Volume 4 Issue 3

Received Date: July 08, 2019

Published Date: July 25, 2019

DOI: $10.23880 /$ cdoaj-16000183

\section{Abstract}

Lymphangioma circumscriptum is a malformation of lymphatic channels which may be localized, generalized, congenital, or acquired, consisting of cysts filled with lymphatic fluid [1]. It may arise anywhere on skin, subcutaneous tissue or mucous membranes [2]. A 47 year- old patient presented with a seventeen-year history of persistent, enlarging, crusted plaques and vesicles, with exudation of clear fluid and blood on her back. This case is reported to present the rare case of adult onset lymphangioma circumscriptum and to present the result of treatment using Carbon dioxide laser as a safe and less invasive option. The favorable result observed in our patient suggests that Carbon dioxide laser may be an additional well-tolerated and safe option for palliation of symptoms in patients with Lymphangioma circumscriptum who are not amenable to surgery.

Keywords: Lymphatic malformation; Lymphangioma circumscriptum; Carbon dioxide laser

\section{Introduction}

Lymphatic malformations are composed of microcysts and/or macrocysts (larger than $1 \mathrm{~cm}$ diameter, previously known as "cystic hygroma" [2]. Lymphangioma circumscriptum, the common form of cutaneous lymphangioma, is characterized by persistent, multiple clusters of translucent vesicles that usually contain clear lymph fluid, resembling frog spawn. The vesicles often are associated with verrucous changes, which give them a warty appearance [3].

\section{Case Report}

A 47 year- old female presented in our institution with multiple clusters of plaques and vesicles filled with clear fluid, topped with yellowish red crusts and erosions located on the left side of back. The lesions were first noted at the age of thirty as few vesicles on erythematous base over the back. No prior history of trauma. Lesions gradually progressed and there was exudation of clear fluid and minimal blood after spontaneous rupture and trauma of vesicles. The lesions also evolved into crusted plaques which eventually became hard and firm scars. Biopsy was done which revealed lymphangioma circumscriptum however, no intervention was done. At the age of thirty- seven, there was noted increase in number of lesions hence she sought consult and six sessions of cryotherapy were done with noted significant improvement. Patient was then lost to follow- up. One month prior, she had undocumented fever lasting for a few days and appearance of new vesicles with some coalescing into bullae on the same site of the back. Her 
past medical history, drug history, social history, and family history were noncontributory.

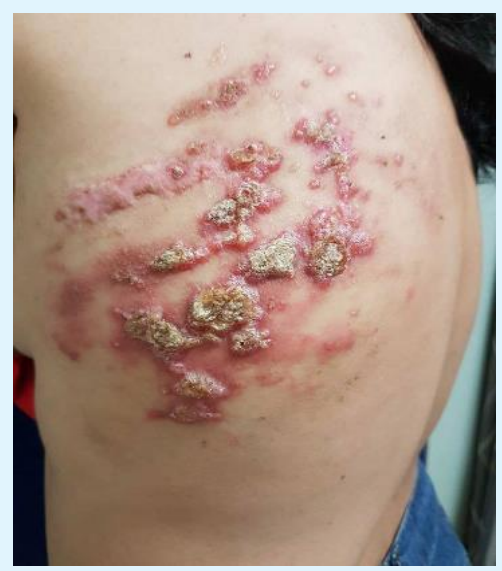

Figure 1: Lymphangioma circumscriptum. Multiple localized, well- circumscribed clusters of plaques and vesicles filled with clear fluid, topped with yellowish red crusts, some eroded, with surrounding erythema, located on the left side of back.
Cutaneous examination revealed multiple wellcircumscribed clusters of plaques and vesicles filled with clear fluid, topped with yellowish red crusts, some eroded, with surrounding erythema, located on the left side of back (Figure 1). The rest of the general physical examination was unremarkable.

The histopathological examination revealed scale crust at the stratum corneum; hyperplastic epidermis with numerous dilated spaces lined by endothelial cells filled with erythrocytes and plasma cells. Lymphatic valve seen. Epidermis is normal while in the dermis, there are moderate capillaries lined by endothelial cells filled with plasma cells, lymphocytes and neutrophils (Figure 2). Ultrasound of the lesion was requested to check for the depth however, the patient has poor compliance. The patient didn't want to undergo other tests, but still willing to have laser done.

A diagnosis of Lymphangioma circumscriptum was made. Patient was referred to General Surgery for further evaluation and management. However, patient refused surgical intervention.

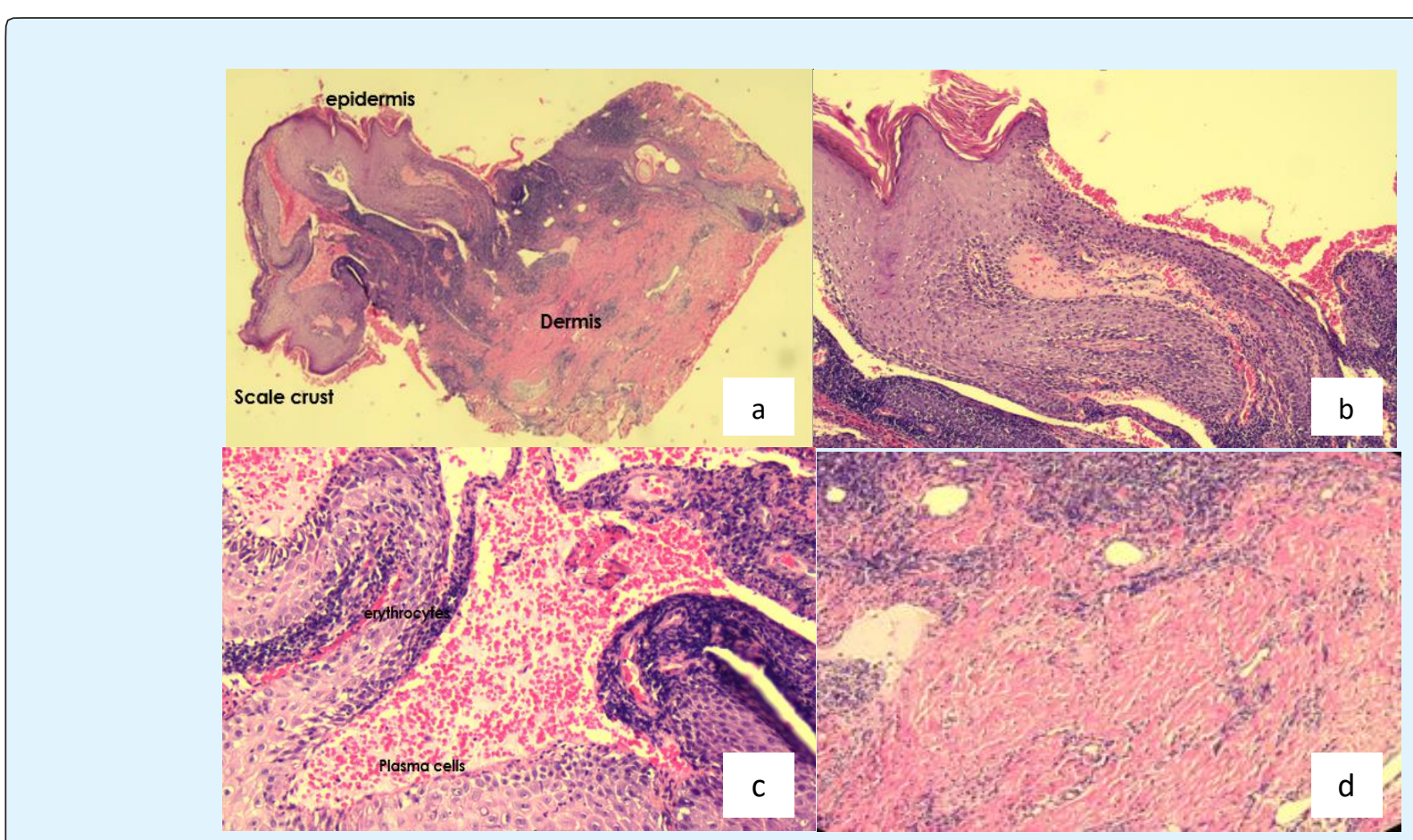

Figure 2: Lymphangioma circumscriptum. a) Hyperplastic epidermis with numerous dilated spaces filled with erythrocytes and plasma cells; scale crust at stratum corneum (40x). b) Dilated spaces are lined by endothelial cells filled with proteinaceous/homogenous material which indicates that these are lymphatic channels (100x). c) Erythrocytes and plasma cells (400x). d) Dermis shows few moderately dilated spaces lined by endothelial cells and filled with homogenous material (400x). 


\section{Discussion}

Lymphangiomas account for $4 \%$ of all vascular tumors, but comprise $25 \%$ of benign vascular growths in children [3]. Equal sex incidences are reported for lymphangiomas in most studies. Lymphangioma can become evident at any age, but the greatest incidence occurs at birth or early in life. About $50 \%$ of lymphangiomas are seen at birth, and most lymphangiomas are evident by the time the patient is aged 5 years [3]. Though it may arise anywhere on skin, subcutaneous tissue or mucous membranes, the common sites include head and neck, oral cavity (tongue), followed by proximal extremities, buttocks, and trunk. It may sometimes be found in penis, vulva, scrotum.

The specific risk factors of lymphangioma circumscriptum are unidentified. In some individuals, it is reported to be acquired due to iatrogenic factors such as surgery to the region and radiation treatment to the area [4].

For the pathogenesis, it is postulated that there is collection of lymphatic cisterns in the deep subcutaneous plane. These cisterns are separated from the normal network of lymph vessels, but they communicate with the superficial lymph vesicles through vertical, dilated lymph channels [5]. A thick coat of muscle fibers that cause rhythmic contractions line these sequestered primitive sacs. Rhythmic contractions increase the intramural pressure, causing dilated channels to protrude from the walls of cisterns toward the skin. Obstruction of lymphatic channels may be caused by inflammatory or infectious pathology. Lymph nodes involved by a malignancy may obstruct the lymphatics by external pressure. These conditions are the underlying causes of localized or acquired lymphangioma circumscriptum [3].

Classical types of Lymphangioma circumscriptum are Lymphangioma simplex which appears as solitary, wellcircumscribed, skin-colored lesion presenting in infancy; Lymphangioma circumscriptum; Cavernous lymphangioma which presents at birth or infancy appearing as large, poorly defined lesion, most commonly involving head, neck, mouth, extremities; and Cystic hygroma which is a type of cavernous lymphangioma with large, deforming cystic lymphatic dilatation [1]. Now, they are broadly grouped into superficial and deep depending upon depth and size of lymphatic channels [1].

Peachy, et al. has further categorized cutaneous lymphangiomas into classic and localized types differing in site, size, and symptoms [6]. The classic form occurs at or shortly after birth, involves an area exceeding $1 \mathrm{~cm}^{2}$ in size and the sites of predilection are proximal extremities, axilla and chest. It is unusual for the classic form to progress from its original vesicular appearance to warty plaques. The localized form involves an area $<1 \mathrm{~cm}^{2}$ in size and it affects any age group and any part of body. There is no definite area of predilection and lesions are asymptomatic [1].

In our patient's case, she could not be classified into either of the two categories (classical or localized). The lesion of our patient first appeared at 30 years of age (localized) and area involved was more than $1 \mathrm{~cm}^{2}$ in diameter (classic). Several reports document the occurrence of late onset lymphangioma circumscriptum (LC) in the setting of lymphatic obstruction [7]. However, our patient had no apparent associated lymphatic abnormalities. Varied clinical presentations of LC are known to occur and lymphangioma in these cases developed following filariasis, surgery or radiation therapy. However, our case was unassociated with any such history. The patient had no significant past medical or surgical history and the cause of the Lymphangioma circumscriptum remain unidentified.

For the treatment, surgical excision has been demonstrated to be superior to other treatment modalities and is effective in removing the primary lesion with a recurrence rate of $17 \%$ to $23 \% 6$. To successfully prevent recurrence, the deep lymphatic cisterns that feed the superficial dilated channels must be removed. Thus, the excision must include the full thickness of the skin, down to deep fascia [8]. For patients in whom surgery is not an option, cauterization, sclerotherapy, cryotherapy and carbon dioxide lasers have been identified as acceptable alternatives for palliative treatment. For all of these modalities, patients should be informed that recurrence is common and should be expected most likely within a year [8].

In our patient, she was referred to General Surgery but she declined surgical intervention hence carbon dioxide laser was done. Patient tolerated the procedure and finished one session of Carbon dioxide laser treatment. After the first session, there was noted improvement with decrease in thickness of plaques, decrease in crusting, and erythema as seen in Figure 3. 


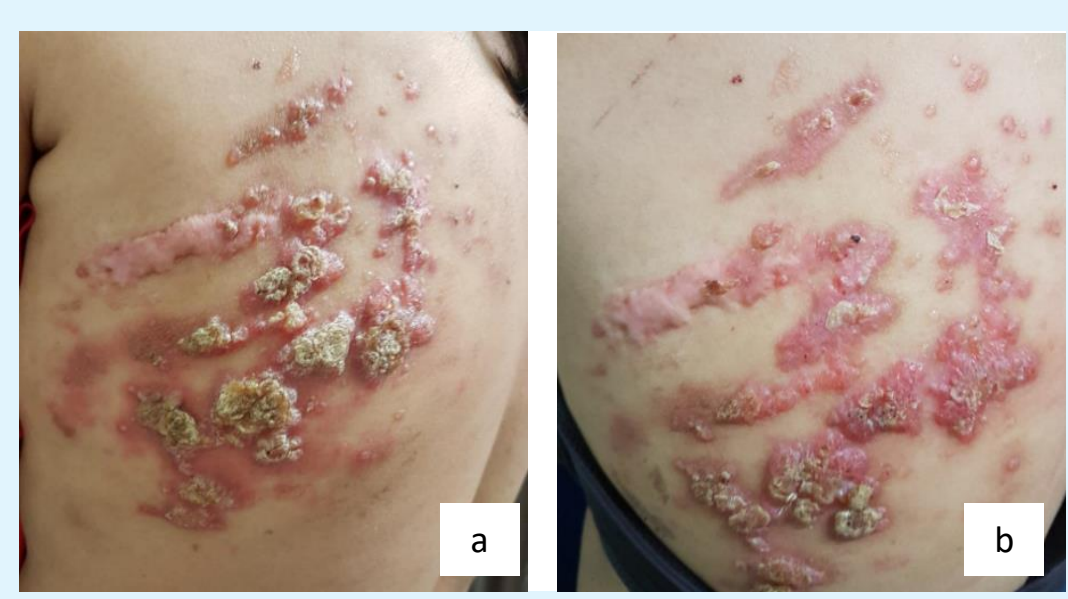

Figure 3: Lymphangioma circumscriptum. a) Baseline image of lesions on the left side of back. b) One week postprocedure using $\mathrm{CO} 2$ laser cutting and ablation with a power setting of 10 watts and a defocused 2.0-mm beam. There was noted decrease in crusting, erythema, and there was flattening of some lesions.

Carbon dioxide laser vaporizes the underlying tissues and seals the lymphatic channels and superficial vascular components. The damage is very localized, with minimal thermal transmission, and scatter. This allows focal destruction with reservation of surrounding and underlying tissue [9]. A study by Savas, et al. concluded that $\mathrm{CO}_{2}$ laser is a safe and efficacious option for the treatment of Lymphangioma circumscriptum, particularly in large lesions that may not be amenable to surgical intervention. Although it is well tolerated, it has the potential for scarring, prolonged erythema, or postinflammatory hyperpigmentation [10]. Another study done by Eliezri and Sklar used $\mathrm{CO}_{2}$ laser in short bursts of continuous output at a power setting of 5 watts in the defocused mode with a $2 \mathrm{~mm}$ spot size. Patients in the study had one session of treatment. The laser beam was used to remove the roof of the vesicles and then to lightly vaporize the floor of the wound. No new vesicles have appeared in any treatment site during 2.5 years after treatment [9]. Early evaluation is needed to prevent recurrent cellulitis which is the major complication. Other complications depend upon location. Prognosis is excellent since lymphangiomas are benign and malignant transformation is rare.

\section{Conclusion}

Lymphangioma circumscriptum is a rare skin lesion that may range from a minute vesicle to a small bullasized lesion which are recurrent. Surgical excision is regarded as the most definitive treatment, giving the highest chance of cure. Other safe and less invasive options are available such as carbon dioxide laser. The favorable result observed in our patient suggests that carbon dioxide laser may be an additional well-tolerated and safe option for palliation of symptoms in patients with Lymphangioma circumscriptum who are not amenable to surgery. But given the high risk of recurrence, a longer- term observation is needed before making definite conclusions [3,11-13].

\section{References}

1. Saira F, Nasir U, Romana I, Minhas K, Ahmad Z, et al(2015) Lymphangioma circumscriptum: Clinicopathological Spectrum of 29 cases. J Coll Physicians Surg Pak 25(9): 658-661.

2. Boon L, Vikkula M (2012) Vascular Malformations. In: Goldsmith L, Katz S, Gilchrest B (Eds.), Fitzpatrick's Dermatology in General Medicine. 8th (Edn.), The McGraw Hill, New York, United States of America, pp: 2086-2087.

3. Schwartz RA, Fernández G (2009) Lymphangioma. eMedicine Dermatology.

4. Kandula S, Lawrence M, Simon W (2012) Acquired Lymphangioma circumscriptum. Journal of American Academy of Dermatology 66(4): AB41.

5. Whimster IW (1976) The Pathology of Lymphangioma Circumscriptum. Br J Dermatol 94(5): 473-486. 


\section{Clinical Dermatology Open Access Journal}

6. Peachey RD, Lim CC, Whimster IW (1970) Lymphangioma of skin: A review of 65 cases. Br J Dermatol 83(5): 519-527.

7. Mohanty S, Gandhi V, Singal A, Baruah MC(1998) Lymphangioma circumscriptum of scrotum of late onset. Indian J Dermatol Venereol Leprol 64(6): 289290.

8. Anderson B, Foulke G (2017) Lymphangioma Circumscriptum. Clinical Advisor.

9. Eliezri Y, Sklar J (1988) Lymphangioma circumscriptum: Review and Evaluation of Carbon Dioxide Laser Vaporization. J Dermatol Surg Oncol 14(4): 357-364.

10. Savas JA, Ledon J, Franca K, Chacon A, Zaiac M, et al. (2013) Carbon Dioxide Laser for the Treatment of
Microcystic Lymphatic Malformations (lymphangioma circumscriptum): a systematic review. Dermatol Surg 39(8): 1147-1157.

11. Puri N (2015) Treatment Options of Lymphangioma Circumscriptum. Indian Dermatol Online J 6(4): 293294.

12. Shumaker PR, Dela Rosa KM, Krakowski AC (2013) Treatment of Lymphangioma Circumscriptum Using Fractional Carbon Dioxide Laser Ablation. Pediatr Dermatol 32(5): 584-586.

13. Saluja S, Peterson M, Summers E (2015) Fractional Carbon Dioxide Laser Ablation for the Treatment of Microcystic Lymphatic Malformations (Lymphangioma Circumscriptum) in an Adult Patient with Klippel- Trenaunay Syndrome. Lasers Surg Med 47(7): 539-541. 